\title{
Efecto del uso de modelos en publicaciones online sobre la generación Electronic Word-of-Mouth
}

\begin{abstract}
Resumen
El propósito de esta investigación consiste en establecer el efecto de la popularidad y el género de los modelos usados en publicaciones de Facebook sobre la generación y/o difusión de Electronic Word of Mouth (eWOM) por parte de usuarios de dicha red social. Para cumplir el objetivo, se analizan 300 publicaciones de marca en Facebook, de las tres empresas colombianas de telefonía móvil con un mayor número de seguidores. Mediante análisis de contenido, se codifican las publicaciones en función del tipo de celebridad que utilizaron y, posteriormente, se aplica un modelo de regresión de Poisson robusta para contrastar las hipótesis propuestas. Los resultados sugieren que el uso de celebridades famosas aumenta la participación de los usuarios por medio del eWOM, mientras que, el uso de modelos comunes disminuye dicha participación. Por otra parte, el uso de modelos masculinos mejora significativamente la tasa de reacciones y contenido compartido con respecto a modelos femeninos. Los hallazgos plantean un aporte a la literatura escasa encargada de analizar la influencia del uso de modelos en publicidad sobre el eWOM, además, plantea recomendaciones gerenciales que pueden ser aplicadas por marcas en sus redes sociales para aumentar la interacción de sus usuarios.
\end{abstract}

\author{
Carlos Fernando Osorio \\ Andrade \\ Magíster en Ciencias de la \\ Organización \\ Profesor Universidad del Valle, \\ Cali, Colombia. \\ Correo electrónico: \\ carlos.fernando.osorio@ \\ correounivalle.edu.co \\ ๑ orcid.org/0000-0002-5095-4991 \\ Google Scholar \\ Claudia Patricia Murcia \\ Zorrilla \\ Doctoranda en Sociedad de la \\ Información y el Conocimiento \\ Profesora Universidad Libre Cali. \\ Correo electrónico: claudiap. \\ murciaz@unilibre.edu.co \\ ๑ orcid.org/0000-0002-5387-9210

\section{Google Scholar}

Augusto Rodríguez Orejuela Doctor en Ciencias de la Empresa Profesor Universidad del Valle, Cali, Colombia.

Correo electrónico:

augusto.rodriguez@correounivalle.edu.co. ๑ orcid.org/0000-0003-2865-1748

Google Scholar

Recibido: junio 14 de 2019

Aprobado: noviembre 03 de 2020

Palabras clave:

estrategia de comunicación, género, marketing, popularidad, telefonía. 


\section{Effect of the use of models in online publications on the generation Electronic Word-of-Mouth}

\begin{abstract}
The purpose of this research is to establish the effects of the popularity and gender of the models used in Facebook posts on the generation and/or the dissemination of Electronic Word of Mouth (eWOM) by users of said social network. To accomplish the objective, 300 brand publications from the three Colombian mobile phone companies with the largest number of followers on Facebook were analyzed. Through content analysis, publications are coded based on the type of celebrity they used and, subsequently, a robust Poisson regression model is applied to test the proposed hypotheses. The results suggest that the use of famous celebrities increases the participation of users through eWOM, while the use of common models decreases this participation. On the other hand, the use of male models significantly improves the rate of reactions and shared content with respect to female models. The findings make a contribution to the scarce literature in charge of analyzing the influence of the use of advertising models on eWOM. In addition, it raises managerial recommendations that can be applied by brands in their social networks to increase the interaction of their users.
\end{abstract}

Key words:

communication strategy, gender, marketing, popularity, telephony. 


\section{Introducción}

La literatura académica sugiere que entre el $25 \%$ y el $30 \%$ de la publicidad que realizan las marcas en países occidentales, hace uso de modelos o personajes, con el objetivo de potenciar los mensajes de marca (Schimmelpfennig, 2018). Con la llegada de Internet y el auge de las redes sociales, las organizaciones hacen uso de modelos en publicaciones online para respaldar sus mensajes comerciales (Demangeot y Broderick, 2010).

De acuerdo con investigaciones científicas, el uso de modelos puede conducir a comportamientos deseados en los consumidores (Garretson y Burton, 2005; Lenoir et al., 2013; Yoon y Kim, 2015; Knoll y Matthes, 2016). Sin embargo, la revisión de literatura en bases de datos indexadas (Scopus y Web of Science), arrojó la ausencia de estudios que establezcan los efectos del uso de modelos en publicidad digital sobre la generación y/o difusión de Electronic Word-ofMouth (eWOM).

El eWOM es una adaptación del voz a voz tradicional a los entornos digitales. Se refiere a la capacidad que tiene el consumidor para realizar cualquier tipo de declaración (positiva, negativa o neutral), acerca de productos y servicios en Internet (Dellarocas, 2003). En redes sociales como Facebook, el eWOM, generalmente, se representa por medio de comentarios, "me gusta", contenido compartido y reacciones (De Vries et al., 2012; Tafesse, 2015; Schultz, 2017).

Con base en la oportunidad de investigación identificada, el propósito de este estudio es aumentar la comprensión de los efectos que ejerce el uso de modelos en publicaciones online; específicamente, sobre la generación y/o difusión de eWOM. Se analizó el papel de Facebook, como mecanismo de promoción en la industria de la telefonía móvil en Colombia; concretamente, se analizaron 300 publicaciones de marca de las empresas Claro, Movistar 
y Tigo (las más representativas en su categoría). La industria de la telefonía móvil fue seleccionada para esta investigación por dos razones. En primer lugar, estas organizaciones tienen un alto número de seguidores en sus redes sociales: según el portal SocialBakers, que mide la reputación de las marcas en medios sociales de todo el mundo, las tres marcas seleccionadas se encuentran dentro del top 15 de empresas con más seguidores en Facebook en Colombia. En segundo lugar, esta categoría de mercado es diferente a las industrias que han sido analizadas por otros estudios sobre eWOM (De Vries et al., 2012; Pletikosa y Michahelles, 2013; Tafesse \& Korneliussen, 2013; Schultz, 2017).

Para identificar y operacionalizar los tipos de modelos utilizados en publicidad, se revisaron los antecedentes de investigación. Se configuraron dos variables independientes: popularidad y género del modelo. La primera se refiere a la fama que tiene el personaje, quien puede ser un individuo común o una celebridad. La segunda refleja si el modelo utilizado es un hombre, una mujer o un personaje sin género definido, como puede ser un "cartoon". Para codificar las categorías propuestas, se utilizó el análisis de contenido

El eWOM se operacionalizó por medio de comentarios, contenido compartido (shares) y reacciones de Facebook; dimensiones que representan la participación de los usuarios en esta red social. Posteriormente, se formularon las hipótesis de investigación que establecen la relación entre el uso de distintos tipos de modelos y la generación de eWOM. Para contrastar estas hipótesis, se aplicó un modelo de regresión generalizada. Los resultados ofrecen modelos significativos que sugieren relaciones importantes entre los distintos tipos de modelos utilizados en publicaciones de Facebook y la generación de eWOM; asimismo, plantean contribuciones interesantes a la literatura y a la gestión de redes sociales en las organizaciones. 


\section{Fundamento teórico}

\section{Electronic Word-Of-Mouth}

La comunicación de boca a oído, o Word-Of-Mouth (WOM), como se dice en el mundo angloparlante, es considerada por los teóricos del marketing como un elemento de gran influencia sobre las actitudes de los consumidores (Engel et al., 1969). Con la llegada de Internet y los medios sociales, este tipo de comunicación (boca a oído) se transforma para adaptarse a los ecosistemas digitales. De esta manera, nace Electronic Word-Of-Mouth (eWOM). Esta forma de comunicación se refiere a la posibilidad que tienen los usuarios de internet para expresar sus opiniones, en cualquier momento y lugar, a través de dispositivos electrónicos, dejando sus comentarios e ideas a disposición de toda una comunidad global de personas (Dellarocas, 2003). Acciones como "dar me gusta" a una publicación, compartir contenido de marca, puntuar o evaluar un servicio y, por supuesto, reaccionar a un mensaje publicitario, son acciones consideradas como eWOM (Schultz, 2017). Para efectos de la presente investigación, que tiene lugar en Facebook, el eWOM será operacionalizado como el volumen de comentarios, reacciones y difusiones que obtiene cada publicación de marca.

En bases de datos académicas como Scopus y Web of Science varios estudios sugieren que, por lo menos, el $60 \%$ de los consumidores participa del eWOM antes de tomar sus decisiones de compra (Li \& Du, 2017; Liu et al., 2018). De hecho, varios autores señalan que el eWOM es la mayor manifestación de lealtad por parte de un cliente satisfecho, o comprometido con una marca u organización (Yeh y Choi, 2011; Casidy y Wymer, 2015).

Vale la pena señalar que cuando los consumidores realizan declaraciones en línea respecto a marcas, productos y servicios, estas declaraciones pueden ser 
positivas o negativas. Investigaciones anteriores en el campo del marketing han determinado que la polaridad de los comentarios influye de manera distinta sobre las actitudes de consumo de las personas (Zhang et al., 2020). Heejae y Dahana (2017), por ejemplo, hallaron que las opiniones positivas influyen de manera significativa en la aceptación de la marca y en el posterior aumento de las ventas. Asimismo, el eWOM que contiene connotaciones negativas sobre los productos y servicios ejerce efectos contrarios sobre estas actitudes deseadas. En otras palabras, la satisfacción - insatisfacción con los productos o servicios y la experiencia con la marca, pueden impulsar la generación y/o difusión de opiniones positivas y negativas, según sea el caso. Estas opiniones, al quedar disponibles para consumidores potenciales, pueden influir sobre el comportamiento de compra (Pérez, 2014).

El papel quejuega eleWOM enel cumplimientodelosobjetivos organizacionales, no ha pasado inadvertido por los investigadores del marketing. En virtud de ello, se han desarrollado múltiples estudios que exploran los antecedentes o factores que impulsan la generación y/o difusión de eWOM (Guo y Zhou, 2017; Li y Du, 2017; Sarmiento et al., 2018). Sin embargo, no se encontraron, hasta el momento, estudios que exploren el uso de modelos en publicidad, como antecedente del eWOM.

\section{Modelos en publicidad}

Los estudios científicos que han analizado los efectos del uso de modelos o personajes en publicidad, sostienen que las características de estos personajes pueden ejercer efectos significativos sobre las actitudes de consumo (Shyong et al., 2013; Lenoir, et al., 2013). Además, aseguran que el uso de modelos puede ser de gran ayuda en la comunicación de beneficios y valores agregados; por esta razón, la elección de modelos para publicidad se convierte en una de las decisiones más importantes para el estratega de marketing (Lynch y Schuler, 
1994). A continuación, se exponen algunas características de los modelos en publicidad, explorados por la literatura académica:

\section{Popularidad del modelo}

En redes sociales, las organizaciones pueden publicar contenido con modelos que refuerzan los mensajes de marca, ya sean personas comunes o celebridades. La literatura académica ha comprobado que el uso de personajes con altos niveles de popularidad, puede incentivar en las personas determinados comportamientos de consumo (Long, 2011; Yoon y Kim, 2015; Kim et al., 2017). Algunas investigaciones — como las de Winterich et al. (2018) han encontrado que la inclusión de personajes famosos en publicidad puede mejorar la actitud de los consumidores hacia productos y servicios y, de tal manera, contribuir a mayor credibilidad para las organizaciones. De manera similar, Derdenger et al. (2018), sostienen que los anuncios con modelos populares, pueden contribuir a aumentar la conciencia sobre la marca, e incluso tener efectos positivos sobre las ventas.

Aunque la mayoría de investigaciones reporta efectos relevantes sobre el uso de modelos famosos en anuncios publicitarios, algunos estudios han indicado que este tipo de modelos, bajo ciertas circunstancias, puede conducir a efectos negativos. Perelló et al. (2018), por ejemplo, aseveran que, en el contexto de la radio, el uso de portavoces desconocidos genera mejores resultados que el uso de celebridades.

El hecho de que los modelos famosos, en general, mejoren la percepción de los consumidores hacia la publicidad, se puede explicar por la dimensión cultural - conocida como creencias de distancia de poder o grado de desigualdad de poder-, que las personas esperan y aceptan en una cultura (Hofstede, 2001; Oyserman 2006). Bajo esta perspectiva, los consumidores pueden respetar y 
"escuchar" con mayor facilidad las opiniones de figuras de autoridad, donde se incluyen las celebridades (Oyserman, 2006). En concordancia con lo que sugieren las creencias de distancia de poder y los antecedentes de investigación, se plantea la siguiente hipótesis:

H1. El uso de celebridades, en anuncios de marca, aumenta la participación de los usuarios de la red social Facebook en forma de eWOM.

\section{Género del modelo}

Dentro del grupo de estudios que explora el papel de los modelos en anuncios publicitarios, existe una línea muy importante que establece el rol que cumple el género del modelo sobre las actitudes de los consumidores. En términos generales, se han encontrado resultados divergentes frente a la efectividad de usar hombres o mujeres en publicidad (Pradhan et al., 2017). Sin embargo, la mayoría de autores parece coincidir en que la congruencia entre el género del modelo y el género del receptor conduce a comportamientos de marca deseados en el público objetivo (Crijns et al., 2017). En otras palabras, la publicidad con modelos masculinos es más efectiva para audiencia masculina; mientras que, la publicidad con modelos femeninas mejora la receptividad de la audiencia de mujeres (Hoffner, 1996; Pileliene y Grigaliunaite, 2017).

Cabe destacar lo siguiente: la correspondencia entre el género del modelo y el receptor no siempre conduce a actitudes de consumo deseadas. Bajo ciertas circunstancias, del medio o del mensaje, la congruencia de géneros puede incidir negativamente en los consumidores. Por ejemplo, Kyrousi et al. (2016), estudiaron la aparición de modelos femeninas en anuncios publicitarios, e hicieron énfasis en la actitud negativa de las consumidoras hacia el estereotipo de la mujer que aparece como objeto sexual en publicidad. Asimismo, algunas investigaciones, en el contexto de la radio, sostienen que el uso de voces 
femeninas, de tonos bajos, en cuñas radiales, genera actitudes favorables hacia los anuncios y la marca, independientemente del género del receptor (Zoghaib, 2017).

En algunas ocasiones, las empresas se valen de otro tipo de personajes para reforzar sus mensajes de marca, los cuales no pueden ser catalogados como masculinos o femeninos. Un ejemplo de esto son los personajes animados o tipo "cartoon" (Chiu et al., 2009). Tal como sucede con los modelos femeninos y masculinos, el propósito de los personajes animados consiste en ayudar a comunicar los valores agregados de productos y servicios (Stout y Moon, 1990).

En términos generales, la literatura ha comprobado que el género del modelo puede interferir en las actitudes que las personas asumen ante la publicidad. Sin embargo, como esta investigación no dispone de una escala para medir la congruencia del género del espectador con el género del modelo presente en las publicaciones de marca analizadas, se propone que:

H2. El género del modelo utilizado en publicaciones de Facebook tiene efectos significativos sobre el eWOM generado por usuarios de la red social.

\section{Metodología}

\section{Descripción de la muestra}

Se realizó muestreo por conveniencia. Se escogieron, para su revisión, las últimas 100 publicaciones de marca en Facebook, de las tres empresas más representativas de la industria de la telefonía móvil en Colombia (Claro, Movistar y Tigo), para una muestra total de 300 publicaciones. 


\section{Recolección de información}

Se utilizó la técnica de minería de datos, con la cual se hizo un raspado de los mismos. Esta técnica permite extraer información de interés desde los servidores API de las redes sociales y otros sitios web (Jones y Waller, 2013). Se utilizaron los lenguajes de programación R y Rstudio. Con estas herramientas, se extrajeron las piezas de comunicación, el número de comentarios, de reacciones y de difusiones (contenido compartido) de cada publicación.

Posteriormente, se empleó análisis de contenido para caracterizar las piezas de comunicación. El análisis de contenido, básicamente, es una técnica de interpretación de textos, ya sean escritos, grabados, pintados, filmados, o de cualquier forma, en los que haya registros de datos (Andréu, 2011). Implica la lectura (textual o visual), como instrumento de recogida de información; sin embargo, debe realizarse de forma sistemática, cuantitativa, objetiva, replicable y válida (Adorno, 2001; Stojanovic et al., 2018).

\section{Codificación de las variables}

El eWOM, que actúa como variable dependiente, se operacionalizó, mediante el recuento de comentarios (comments), contenido compartido (shares) y reacciones (reacts). Estas métricas se obtuvieron con minería de datos. Para la popularidad del modelo, se diseñó una variable de naturaleza politómica de tres (3) niveles que se codificaron como: (1) las piezas de comunicación que no presentaban personaje alguno, (2) publicaciones donde aparecía un modelo común y (3) cuando se presentaba una celebridad, asumiendo a (1) como categoría de base. Por otro lado, la variable género del modelo, se constituyó con cuatro categorías: (1) no uso de personaje, (2) personaje masculino, (3) personaje femenino y (4) personaje de género no identificado como un cartoon, también incluyendo a (1), como categoría de base. 
En la operacionalización de las variables participaron dos estudiantes de posgrado quienes actuaron como codificadores y fueron previamente entrenados. Estas personas caracterizaron las piezas de comunicación de acuerdo a las tipologías propuestas según los criterios presentados en la Tabla 1.

Tabla 1. Tipologías de las piezas de comunicación

\begin{tabular}{|c|c|c|}
\hline Variable & Operacionalización & Detalles \\
\hline \multirow{3}{*}{$\begin{array}{l}\text { Popularidad } \\
\text { del personaje }\end{array}$} & $\begin{array}{l}\text { Publicación sin personaje (1) } \\
\text { Categoría de base }\end{array}$ & $\begin{array}{l}\text { Aquellas piezas de comunicación en las que no se presentaba ningún } \\
\text { personaje, fueron codificadas como } 1 .\end{array}$ \\
\hline & Personaje común (2) & $\begin{array}{l}\text { Las publicaciones que utilizaron modelos comunes o no } \\
\text { reconocidos, se codificaron como } 2 \text {. }\end{array}$ \\
\hline & Celebridad (3) & $\begin{array}{l}\text { Publicaciones que emplearon un personaje famoso para reforzar sus } \\
\text { mensajes, se codificaron como } 3 \text {. }\end{array}$ \\
\hline \multirow{4}{*}{$\begin{array}{l}\text { Género del } \\
\text { modelo }\end{array}$} & $\begin{array}{l}\text { Publicación sin personaje (1) } \\
\text { Categoría de base }\end{array}$ & $\begin{array}{l}\text { Nuevamente, se codificaron como 1, las piezas en las que no se } \\
\text { presentaban personajes. }\end{array}$ \\
\hline & Modelo hombre (2) & Piezas en las que aparece un hombre, se codificaron como 2. \\
\hline & Modelo mujer (3) & $\begin{array}{l}\text { Piezas de comunicación en las que se presenta una mujer, fueron } \\
\text { codificadas como (3). }\end{array}$ \\
\hline & Modelo sin género definido (4) & $\begin{array}{l}\text { Piezas que presentan personajes sin género definido, fueron } \\
\text { clasificadas como (4). }\end{array}$ \\
\hline
\end{tabular}

Fuente: elaboración propia.

A continuación, en la Figura 1, se aprecia el modelo de investigación para este estudio: 


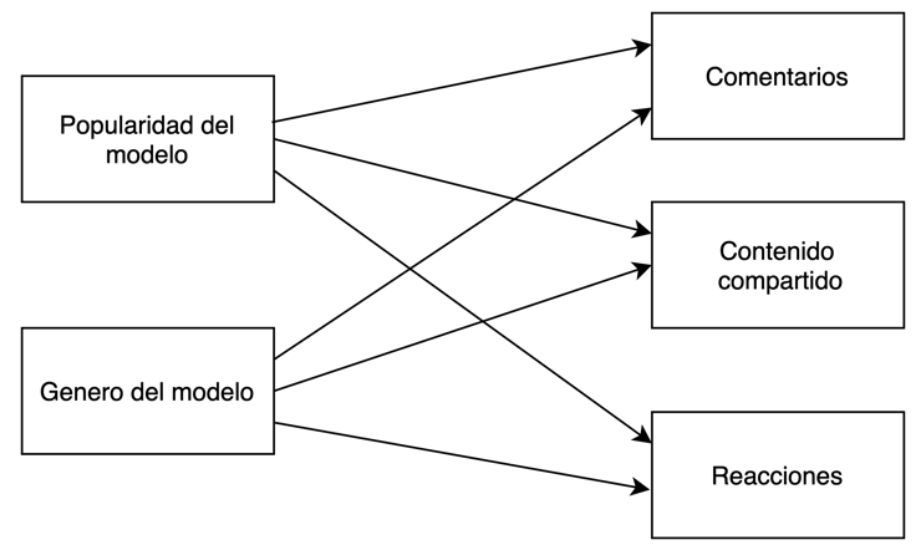

Figura 1. Modelo de investigación. Fuente: elaboración propia.

\section{Fiabilidad de la codificación}

Para lograr la fiabilidad en la operacionalización de las variables, dos estudiantes de posgrado fueron entrenados como codificadores. Estas personas se capacitaron para la comprensión de las definiciones conceptuales y operativas de cada una de las variables incluidas en el modelo. Se llevaron a cabo varias sesiones de capacitación y se modificó constantemente la plantilla de codificación hasta alcanzar un indicador de fiabilidad satisfactorio entre los codificadores. Se eligieron 90 publicaciones de marca (30\% del total) para verificar la consistencia de la operacionalización. Las puntuaciones de fiabilidad de las variables fueron pertinentes, ya que todas ofrecieron estadísticos por encima de 0,80, en el alfa de Krippendorff (2012). 


\section{Análisis empírico}

Para este estudio, la variable dependiente (eWOM) se operacionalizó por medio de sus dimensiones: comentarios, reacciones y difusiones; todas procedentes de un recuento. Las variables de esta naturaleza no deben ser sometidas a modelos de regresión lineal por mínimos cuadrados ordinarios (MCO), porque se viola el supuesto de normalidad. Concretamente, el conjunto de datos empleado presentó asimetría positiva. Para solucionar este inconveniente, un modelo de regresión de Poisson puede ser más adecuado, en la medida que trata con la naturaleza discreta de la variable dependiente (Cameron y Trivedi, 2013).

Un problema frecuente, cuando se utiliza el modelo de regresión de Poisson, es la sobredispersión, que conduce a malinterpretaciones de los errores estándares de los coeficientes, lo que, a su vez, puede derivar en la significancia estadística de factores que no van dentro del modelo (Navarro et al., 2001). Para probar si ocurría sobredispersión en el modelo, se realizó el test de bondad de ajuste de Pearson. Como los estadísticos obtenidos fueron mayores a cero, se asumió la existencia de sobredispersión y se aplicó un modelo con errores robustos, para tratar el problema (Abitbol y Lee, 2017).

De esta forma, se constituyó la ecuación 1:

$$
\operatorname{Ln}(Y)=\propto+\sum_{i=2}^{3} \beta 1, i \text { Pop }_{i}+\sum_{i=2}^{4} \beta 2, j \text { Gen }_{j}
$$

Donde: Ln (Y) corresponde al logaritmo natural de la variable dependiente que puede asumir uno de tres valores: comentarios, reacciones o contenido compartido. a representa el término constante. Pop simboliza la variable 
popularidad del modelo, la cual puede asumir uno de tres valores posibles: (1) no uso de personaje, (2) personaje común, (3) personaje famoso; en este caso, el no uso de personaje (1) se eligió como categoría de base o comparación. Gen es la variable politómica que representa el tipo de personaje que utiliza la publicación y, asume uno de cuatro valores: (1) no uso de personaje, (2) hombre, (3) mujer, o (4) género no definido. Para este caso, también se eligió el no uso de personaje (1), como categoría de base o comparación.

\section{Resultados}

Se analizó un total de 300 publicaciones de marca de las tres empresas de telefonía móvil más representativas de Colombia. La cantidad promedio de publicaciones —muestreada por compañía- fue de 100. Entre las publicaciones, el número promedio de comentarios por cada una fue de 23; el de contenido compartido fue de 14 y el de reacciones fue de 65.

En relación con las categorías analizadas, un 30\% de las publicaciones no utilizó ningún tipo de personaje. Un 53\% de las piezas usó modelos comunes, mientras que un $16 \%$ se valió de personajes famosos para reforzar sus mensajes de marca. En relación con el género de los modelos, un 33\% de las publicaciones empleó un personaje hombre, un 20\% utilizó mujeres y un 13\% usó personajes de género no definido; por ejemplo: personajes animados, animales o "cartoons". En la Tabla 2, se aprecian las estadísticas descriptivas. 
Tabla 2. Estadística descriptiva

\begin{tabular}{|c|c|c|c|c|c|}
\hline eWOM & & Media & SD & Mínimo & Máximo \\
\hline Comentarios & & $23,9 \%$ & 65,52 & 1 & 276 \\
\hline Contenido compartido (shares) & & $14 \%$ & 75,89 & 0 & 1.067 \\
\hline Reacciones & & $65,5 \%$ & 178,99 & 3 & 2.368 \\
\hline \multicolumn{6}{|l|}{ Variables categóricas } \\
\hline & Frecuencia & \multicolumn{4}{|c|}{ Frecuencia-R } \\
\hline No uso personaje & 90 & \multicolumn{4}{|c|}{$30 \%$} \\
\hline Personaje común & 160 & \multicolumn{4}{|c|}{$53.3 \%$} \\
\hline Personaje famoso & 50 & \multicolumn{4}{|c|}{$16.6 \%$} \\
\hline Hombre & 103 & \multicolumn{4}{|c|}{$33 \%$} \\
\hline Mujer & 61 & \multicolumn{4}{|c|}{$20.3 \%$} \\
\hline Género no definido & 40 & \multicolumn{4}{|c|}{$13.3 \%$} \\
\hline
\end{tabular}

Fuente: elaboración propia.

En la Tabla 3, se aprecian los resultados del Modelo de Regresión de Poisson, con errores robustos. Lo primero que se observa es que el modelo para comentarios es marginalmente significativo: el estadístico Wald, con una distribución de $X^{2}$ y 5 grados de libertad, arroja una probabilidad de 0,0661 y ninguna de las variables propuestas es significativa. Respecto a los modelos para contenido compartido y reacciones, ambos son conjuntamente significativos: arrojan una probabilidad de 0,0001 en contenido compartido y 0,0000, en reacciones.

En la variable contenido compartido, puede observarse que las publicaciones que utilizan personajes comunes son marginalmente significativas, con un $90 \%$ de confianza y un signo negativo $\left(\beta=-0,760^{*}\right)$. Esto indica que, cuando se usa este tipo de modelos, la tasa de contenido compartido disminuye 
0,4 (IRR) veces, respecto a los anuncios en los que no aparece ningún tipo de personaje.

En contraste, el uso de personajes famosos presenta significancia estadística: $95 \%$ de confianza y un signo positivo $\left(\beta=1,071^{* *}\right)$. Con el uso de celebridades, la tasa de contenido compartido aumenta 2,9 (IRR) veces, respecto a las piezas que prescinden de modelos. En relación con el género del modelo, el uso de personajes femeninos y de género no determinado no tiene significancia estadística. El uso de personajes masculinos es marginalmente significativo, con un nivel de confianza del $90 \%$, y un signo positivo $\left(\beta=0,977^{*}\right)$. En otras palabras, con el uso de modelos masculinos, en publicaciones de Facebook, la tasa de contenido compartido aumenta 2,6 (IRR) veces, en comparación con la categoría de base.

En la variable dependiente reacciones, el uso de personajes comunes tiene una significancia estadística del $95 \%$ de confianza, con signo negativo $\left(\beta=-0,576^{* *}\right)$, lo que quiere decir que, cuando se usan personajes comunes, la tasa de reacciones en publicaciones disminuye 0,5 (IRR) veces, en relación con las piezas que no usan modelos. En tanto que el uso de celebridades presenta significancia del $95 \%$ y un signo positivo $\left(\beta=0,680^{* *}\right)$; esto significa que, ante el uso de personajes famosos, la tasa de reacciones aumenta 1,9 (IRR) veces, en relación con la categoría de base. Frente al género del modelo, nuevamente el uso de personajes femeninos y de género no determinado presenta insignificancia estadística. No obstante, el uso de personajes masculinos es significativo: $95 \%$ de confianza y un signo positivo $\left(\beta=0,700^{* *}\right)$; es decir que, ante el uso de este tipo de personajes, la tasa de reacciones será 2 (IRR) veces superior, en contraste con las publicaciones que no usan modelos. 
Tabla 3. Modelo empírico

\begin{tabular}{|c|c|c|c|c|c|c|c|}
\hline \multirow{2}{*}{\multicolumn{2}{|c|}{ Variables dependientes }} & \multicolumn{2}{|c|}{ Comentarios } & \multicolumn{2}{|c|}{ Contenido compartido } & \multicolumn{2}{|c|}{ Reacciones } \\
\hline & & $\beta$ & IRR & $\beta$ & IRR & $\beta$ & IRR \\
\hline \multirow{3}{*}{$\begin{array}{l}\text { Popularidad del } \\
\text { modelo }\end{array}$} & Sin personaje (Base) & - & - & - & - & - & - \\
\hline & Personaje común & $-0,164$ & 0,467 & $-0,760^{*}$ & $0,467^{*}$ & $-0,576^{* *}$ & $0.561 * *$ \\
\hline & Celebridad & 0,221 & 2,921 & $1,071^{* *}$ & $2,920^{* *}$ & $0,680^{* *}$ & $1.97^{* *}$ \\
\hline \multirow{4}{*}{ Género modelo } & Sin personaje (Base) & - & - & - & - & - & - \\
\hline & Hombre & $-0,252$ & 2,657 & $0,977^{*}$ & $2,657^{*}$ & $0,700^{* *}$ & $2.013^{* *}$ \\
\hline & Mujer & 0,122 & 0,715 & $-0,334$ & 0,715 & 0,145 & 1.156 \\
\hline & $\begin{array}{l}\text { Género no } \\
\text { determinado }\end{array}$ & 0,346 & 0,802 & $-0,220$ & 0,802 & $-0,743$ & 0.928 \\
\hline \multicolumn{2}{|l|}{ Wald $X^{2}(5)$} & \multicolumn{2}{|c|}{10,33} & \multicolumn{2}{|c|}{26,89} & \multicolumn{2}{|c|}{33,91} \\
\hline \multicolumn{2}{|l|}{ Prob $>X^{2}$} & \multicolumn{2}{|c|}{0,0661} & \multicolumn{2}{|c|}{0,0001} & \multicolumn{2}{|c|}{0,0000} \\
\hline \multicolumn{2}{|l|}{ Pseudo R² \% } & \multicolumn{2}{|c|}{$2 \%$} & \multicolumn{2}{|c|}{$25 \%$} & \multicolumn{2}{|c|}{$21 \%$} \\
\hline
\end{tabular}

${ }^{* * *} \mathrm{p}<0.01,{ }^{* *} \mathrm{p}<0.05,{ }^{*} \mathrm{p}<0.1, \mathrm{IRR}=$ tasa de incidencia.

Fuente: elaboración propia.

\section{Conclusiones}

A partir del estudio realizado acerca del Efecto del uso de modelos en publicaciones online sobre la generación Electronic Word-of-Mouth, es posible, a modo de reflexión, anotar que:

En términos generales, el uso de modelos en publicidad, sin tener en cuenta qué tipo de celebridad se emplea, aumenta el contenido compartido y las reacciones. Sin embargo, todo parece indicar que el uso de personajes en publicaciones de marca no aumenta el número de comentarios. 
Aunque las métricas mencionadas anteriormente forman parte del mismo constructo y se esperarían resultados similares en cada una de ellas, la Teoría de las Actividades en Línea Relacionadas con la Marca sugiere que diversas acciones pueden implicar distintos gastos cognitivos y niveles de implicación con la marca (Mutinga et al., 2011). Por ejemplo, ver una publicación o reaccionar ante ella, requiere menor procesamiento cognitivo y menor implicación que comentar en publicaciones de marca o descargar las imágenes al ordenador o el dispositivo móvil (Mutinga, et al., 2011). En este sentido, se podría pensar que el uso de modelos en publicaciones ejerce efectos mayores sobre las acciones de eWOM que implican un menor esfuerzo por parte del espectador.

Los resultados de este estudio indican que el uso de modelos — con altos niveles de popularidad - mejora significativamente la tasa de contenido compartido y las reacciones. Estos hallazgos soportan lo que exteriorizan las creencias de distancia de poder (Hofstede, 2001): los consumidores tendrán mayor predisposición a recibir mensajes de marca, cuando vienen acompañados por figuras de autoridad; en este caso por celebridades.

Respecto al efecto del género del modelo sobre el eWOM, los resultados de este estudio apuntan a que ni los personajes femeninos ni los personajes de género no determinado, como personajes animados y animales, ejercen influencia sobre la tasa de comentarios, contenido compartido y las reacciones. Sin embargo, el uso de personajes masculinos en publicaciones de marca, sí aumenta de manera importante el número de reacciones y contenido compartido por usuarios en redes sociales. Lo anterior puede explicarse dado que, en Latinoamérica, y muy especialmente en Colombia, aún predomina el imaginario social del "machismo". En efecto, existe la creencia generalizada en la superioridad del hombre y, tanto el hombre como la mujer, creen firmemente en dicha "superioridad" masculina (Stycos, 1958; Giraldo, 1972). 
La publicidad ha sido un elemento que históricamente ha contribuido a mantener el imaginario de superioridad masculina en el colectivo social, y no solo en países en vía de desarrollo (McArthur y Resko, 1975). Frecuentemente, el hombre se presenta, en los medios, como el consumidor experto; mientras que la mujer se muestra como una persona más ingenua y, en ocasiones, solamente se explota su atractivo sexual (McArthur y Resko, 1975). Por todo lo anterior, es comprensible que los consumidores, hombres y mujeres, tengan mayor disposición a aceptar y respetar mensajes de marca acompañados de modelos masculinos. Por este motivo, las organizaciones deberían incluir, con mayor frecuencia, personajes masculinos en sus publicaciones de marca, para aumentar la participación de los usuarios en sus redes sociales.

\section{Recomendaciones}

En términos prácticos, las marcas deberían considerar la inversión, por lo menos ocasional, en personajes famosos para ser incluidos en publicaciones de redes sociales, puesto que el uso de modelos comunes, en lugar de aumentar las acciones de eWOM, disminuye significativamente la tasa de contenido compartido y las reacciones. Relacionando este hecho con las creencias de distancia de poder, los consumidores parecen no tener una buena disposición para recibir mensajes de marca, cuando son acompañados por figuras que no ostentan autoridad alguna (Hofstede, 2001). Así que las organizaciones no deberían preocuparse demasiado por invertir en modelos comunes que acompañen sus piezas de comunicación. Por el contrario, el presupuesto destinado para este rubro debería conservarse y usarse exclusivamente en la contratación de celebridades. Claro está que los resultados de esta investigación se enmarcan en el contexto de una industria específica (telefonía móvil en Colombia); por tanto, si se pretenden generalizar las recomendaciones aquí expuestas, deberían realizarse estudios complementarios en otras categorías de productos y servicios. 
Por otro lado, es un hecho que las redes sociales difieren en sus propósitos así como en los públicos objetivos que atienden. Por ende, posiblemente, un usuario frecuente de Facebook puede diferenciarse fácilmente de uno de Twitter, Instagram o la recientemente popular TikTok. En este sentido, futuras investigaciones podrían establecer los efectos del uso de modelos en publicaciones en el espacio de otras redes sociales, o incluso plantear posibles efectos de interacción, o moderación de las diferentes redes.

Finalmente, futuras investigaciones podrían incluir otras características de los modelos que se escapan en el presente estudio; por ejemplo: la raza de los personajes, el atractivo físico y el atractivo sexual de los modelos. Estas son categorías muy interesantes que se pueden explorar en el futuro.

\section{Referencias}

Abitbol, A. y Lee, S. (2017). Messages on CSR-dedicated Facebook pages: What works and what doesn't. Public Relations Review, 43(4), 796-808. https://bit.ly/2WHoOJr

Adorno, T. (2001). Epistemología y Ciencias Sociales. Madrid: Cátedra.

Andréu Abela, J. (2011). Las técnicas de análisis de contenido: una revisión actualizada. Sevilla: Fundación Centro de Estudios Andaluces.

Cameron, A. y Trivedi, P. (2013). Regression analysis of count data. Cambridge: Cambridge University Press.

Casidy, R. y Wymer, W. (2015). The impact of brand strength on satisfaction, loyalty and WOM: An empirical examination in the higher education sector. Journal of Brand Management, 22(2), 117-135. https://bit.ly/2XJlaoR

Chiu, Y., Lin, C., \& Liu, W. (2009). The affect transfer effect on spokes-characters. In ACME Proceedings (pp. 386-98). 
Crijns, H., Claeys, A., Cauberghe, V. y Hudders, L. (2017). Who says what during crises? A study about the interplay between gender similarity with the spokesperson and crisis response strategy. Journal of Business Research, 79, 143-151. https://bit.ly/2F5R9s8

De Vries, L., Gensler, S. y Leeflang, P. (2012). Popularity of Brand Posts on Brand Fan Pages: An Investigation of the Effects of Social Media Marketing. Journal of Interactive Marketing, 26(2), 83-91. https://bit.ly/2jckeGR

Dellarocas, C. (2003). The Digitization of Word of Mouth: Promise and Challenges of Online Feedback Mechanisms. Management Science, 49(10), 1407-1424. https://bit.ly/2F8rtei

Demangeot, C. y Broderick, A. (2010). Consumer perceptions of online shopping environments: A gestalt approach. Psychology and Marketing, 27(2), 117-140. https://bit.ly/2Xa4M3K

Derdenger, T., Li, H. y Srinivasan, K. (2018). Firms' Strategic Leverage of Unplanned Exposure and Planned Advertising: An Analysis in the Context of Celebrity Endorsements. Journal of Marketing Research, 55(1), 14-34. https://bit.ly/2XdA8X5

Engel, J., Kegerreis, R. y Blackwell, R. (1969). Word-of-Mouth Communication by the Innovator. Journal of Marketing, 33(3), 15-19. https://bit.ly/31rv14Z

Garretson, J. y Burton, S. (2005). The Role of Spokescharacters as Advertisement and Package Cues in Integrated Marketing Communications. Journal of Marketing, 69(4), 118-132. https://bit.ly/2RgxcU5

Giraldo, O. (1972). El machismo como fenómeno psicocultural. Revista Latinoamericana de Psicología, 4(3), 295-309. https://bit.ly/2ZqkGnX 
Guo, B. y Zhou, S. (2017). What makes population perception of review helpfulness: an information processing perspective. Electronic Commerce Research, 17(4), 585-608. https://bit.ly/2wNLG4D

Heejae, S., \& Dahana, W. D. (2017). The moderating roles of prior attitude and message acceptance in electronic word of mouth. International Journal of Business and Information, 12(2), 183.

Hoffner, C. (1996). Children's wishful identification and parasocial interaction with favorite television characters. Journal of Broadcasting and Electronic Media, 40(3), 389-402. https://bit.ly/2Fa8W19

Hofstede, G. (2001). Culture's consequences: Comparing values, behaviors, institutions, and organizations across nations (2a ed). London: SAGE Publication.

Jones, J. y Waller, N. (2013). Computing confidence intervals for standardized regression coefficients. Psychological Methods, 18(4), 435-453. https://bit. ly/2F97f47

Kim, J., Lee, J. y Chung, Y. (2017). Product Type and Spokespersons in Native Advertising - The Role of Congruency and Acceptance. Journal of Interactive Advertising, 17(2), 109-123. https://bit.ly/2IcLW3n

Knoll, J. y Matthes, J. (2016). The effectiveness of celebrity endorsements : a meta-analysis. Journal of the Academy of Marketing Science. http://dx.doi. org/10.1007/s11747-016-0503-8

Kyrousi, A., Panigyrakis, G. y Panopoulos, A. (2016). Attitudes toward ads portraying women in decorative roles and female competition: An evolutionary psychology perspective. International Journal of Advertising, 35(5), 771-798. https://bit.ly/2WGd7MU 
Krippendorff, K. (2012). Content analysis: an introduction to its methodology. Los Angeles, CA: SAGE.

Lenoir, A., Puntoni, S., Red, A. y Verlegh, P. (2013). The impact of cultural symbols and spokesperson identity on attitudes and intentions. International Journal of Research in Marketing, 30(4), 426-428. https://bit.ly/2RgPHYH

Li, F. y Du, T. (2017). The effectiveness of word of mouth in offline and online social networks. Expert Systems with Applications, 88, 338-351. https:// doi.org/10.1007/s11747-016-0503-8

Liu, S. Q., Ozanne, M., \& Mattila, A. S. (2018). Does expressing subjectivity in online reviews enhance persuasion? Journal of Consumer Marketing, 35(4),403-413. https://bit.ly/39MiyPN

Long, L. (2011). The impact of advertising appeals and advertising spokespersons on advertising attitudes and purchase intentions. African Journal of Business Management, 5(21), 8446-8457. https://bit.ly/2XeQoXK

Lynch, J. y Schuler, D. (1994). The matchup effect of spokesperson and product congruency: A schema theory interpretation. Psychology \& Marketing, 11(5), 417-445. https://bit.ly/2IfXmnk

McArthur, L. y Resko, B. (1975). The Portrayal of Men and Women in American Television Commercials. The Journal of Social Psychology, 97(2), 209-220. https://bit.ly/2XH1XUG

Mutinga, D., Moorman, M. y Smit, E. (2011). Introducing COBRAs. Exploring motivations for brand-related social media use. International Journal of Advertising, 30(1), 13-46. https://bit.ly/2K3ok0t

Navarro, A., Utzet, F., Puig, P., Caminal, J. y Martín, M. (2001). La distribución binomial negativa frente a la de Poisson en el análisis de fenómenos recurrentes. Gaceta Sanitaria, 15(5), 447-452. https://bit.ly/2IfOePp 
Oyserman, D. (2006). High Power, Low Power, and Equality: Culture Beyond Individualism and Collectivism. Journal of Consumer Psychology, 16(4), 352-356. https://bit.ly/2X6vvhD

Perelló, S., Muela, C. y Campos, M. (2018). La personificación de la marca en la publicidad radiofónica. Análisis de la presencia y uso de la figura del portavoz. Revista Latina de Comunicación Social (73), 1163-1178. https://bit.ly/2MHZE2v

Pérez, M. L. (2014). Marketing boca a boca. Cómo conseguir que el consumidor hable de tu producto o marca (Tesis de doctorado). Departamento de Comercialización e Investigación de Mercados, Universidad de Murcia Facultad de EconomíaY Empresa. http://www.tdx.cat/handle/10803/144666

Pileliene, L. y Grigaliunaite, V. (2017). The effect of female celebrity spokesperson in FMCG avertising: neuromarketing approach. Journal of Consumer Marketing, 34(3), 202-213. https://bit.ly/2 lelUfi

Pletikosa, I. y Michahelles, F. (2013). Online engagement factors on Facebook brand pages. Social Network Analysis and Mining, 3(4), 843-861. https://bit.ly/2FHKsiJ

Pradhan, D., Kapoor, V. y Moharana, T. (2017). One step deeper: gender and congruity in celebrity endorsement. Marketing Intelligence \& Planning, 35(6), 774-788. https://bit.ly/2XNTsr8

Sarmiento, J., Curiel, J. y Antonovica, A. (2018). Word-of-Mouth Communication as a Consequence of Relationship Quality in Online Environments. Palabra Clave, 21(4), 1075-1106. https://bit.ly/2IE1gVX

Schimmelpfennig, Ch. (2018). Who is the Celebrity Endorser? A Content Analysis of Celebrity Endorsements Who is the Celebrity Endorser? A Content Analysis of Celebrity Endorsements. Journal of International Consumer Marketing, 30(4), 220-234. https://bit.ly/2KKRzrv 
Shyong, J., Cheng, Y. y Chu, Y. (2013). Effect of Celebrity Endorsements on Consumer Purchase Intentions: Advertising Effect and Advertising Appeal as Mediators. Human Factors and Ergonomics in Manufacturing, 23(5), 357-367. https://bit.ly/2MMy2tk

Schultz, C. (2017). Proposing to your fans: Which brand post characteristics drive consumer engagement activities on social media brand pages? Electronic Commerce Research and Applications, 26, 23-34. https://bit. ly/2WF1HUK

Stycos, J. (1958). Familia y fecundidad en Puerto Rico; estudio del grupo de ingresos más bajos. México, D.F.: Fondo de Cultura Económica.

Stojanovic, I., Andreu, L. y Curras, R. (2018). Effects of the intensity of use of social media on brand equity: An empirical study in a tourist destination. European Journal of Management and Business Economics, 27(1), 83-100. https://bit.ly/2JYDEOw

Stout, P.yMoon,Y.(1990). Useofendorsersinmagazineadvertisements.Journalism \& Mass Communication Quarterly, 67(3), 536-546. https://bit.ly/2RcTNB2

Tafesse, W. (2015). Content strategies and audience response on Facebook brand pages. Marketing Intelligence and Planning, 33(6), 927-943. https://bit.ly/2XaEs9n

Tafesse, W. y Korneliussen, T. (2013). Examining the effect of using multiple media tools on the marketing performance of organizations in a trade campaign environment. Journal of Marketing Communications, 19(3), 215-227. https://bit.ly/2Xepyz3

Winterich, K., Gangwar, M. y Grewal, R. (2018). When Celebrities Count: Power Distance Beliefs and Celebrity Endorsements. Journal of Marketing, 82(3), 70-86. https://bit.ly/2ZmzHah 
Yeh, Y. y Choi, S. (2011). Mini-lovers, maxi-mouths: An investigation of antecedents to eWOM intention among brand community members. Journal of Marketing Communications, 17(3), 145-162. https://bit.ly/2WINtXZ

Yoon, D. y Kim, Y. (2015). The Roles of a Spokesperson and Brand-Message Congruity in Advertising Effectiveness of Coffeehouse Brands The Roles of a Spokesperson and Brand-Message Congruity in Advertising. Journal of Quality Assurance in Hospitality \& Tourism, 16(4), 347-368. https://bit.ly/2ZmMlke

Zhang, H., Liang, X., \& Qi, C. (2020). Investigating the impact of interpersonal closeness and social status on electronic word-of-mouth effectiveness. Journal of Business Research. https://www.x-mol.com/ paperRedirect/1347277775796588544

Zoghaib, A. (2017). The contribution of a brand spokesperson's voice to consumer-based brand equity. Journal of Product \& Brand Management, 26(5), 492-502. https://bit.ly/2X6vQAT

Cómo citar: Osorio Andrade C. F., Murcia Zorrilla, C. P., Rodríguez Orejuela, A. (2021). Efecto del uso de modelos en publicaciones online sobre la generación Electronic Word-of-Mouth. Revista KEPES, 18(23), 375-400. https://doi.org/10.17151/kepes.2021.18.23.13 